\title{
Stability constants and stoichiometries of chromium and zirconium carboxylates complexes calculated by four comparative methods
}

\author{
Abdalazeem A. Omar *, Elmugdad A. Ali \\ Department of chemistry, faculty of science, Sudan University of Science and Technology, Sudan \\ *Corresponding author E-mail: abdeenomar78@hotmail.com
}

\begin{abstract}
Four comparative methods for determination of stoichiometries and stability constants of chromium and zirconium complexes with citric and propanoic acids were studies, in purpose to select the most accurate and sensitive method, and to compare the formation of complexes of these two transition metals. (Cr 1st transition series group 6B, $\mathrm{Zr}$ 2nd transition series group 4B)

Metal-ligand stability constants and stoichiometries of the above systems were determined by Calvin \& Bjerrum pH-metric titration technique as adopted by Irving \& Rossotti.

Four methods employed were point-wise calculation method, half integral method, linear plot method and least squares method. The result seems in a good agreement with each other and any method can represents the other.
\end{abstract}

Keywords: Half integral method; Henderson equation; Stability constants; Excel program; activity.

\section{Introduction}

Complexation studies are very important from the point of view of analytical and biochemical researchers, the extent to which the ligand bind to a metal ion is expressed in terms of stability constants (Sanjay et al, 2002). Dissociation of organic acid and their interactions with metal ions (complex formation) may be extremely sensitive to ionic strength of the medium (Santosh et al, 2011). Studies in matetal-ligand complexes in a solution of a number of metal ions with carboxylic acids, oximes, phenols etc. would interestingly throw a light on the mode of storage and transport of metal ions in biological kingdom (Summer et al, 2011). If the stability constant of a reaction between metal and ligand has found greater than zero, is perhaps one of the most convincing evidence for the existence of the complex species (Abdulbaset et al. 2012). The equilibrium constants that refer to the interaction of metal cations and ligands are widely referred to as stability constants. In practice there are two types of stability, mainly thermodynamic and kinetic (Hartley et al. 1980). The main transition metal groups or d-block includes these elements that have partially filled $\mathrm{d}$-shells, thus chromium is half-filled 3d- shells (Cr: [Ar] 4s13d5) and zirconium is partially filled 4d-shells, (Zr: [Kr] 5s2 4d2) (Cotton et al 1972, Boner et al 1996).

Citric acid is weak organic acid with formula $\mathrm{C} 6 \mathrm{H} 8 \mathrm{O} 7$ a white crystalline powder with molar mass 192.124.It consists of three carboxylic groups. (http://en.wikipedia.org/wiki/Citric_acid. 2014) Propanoic acid also known as propionic acid from Creek words protos meaning first and pion meaning fat. It is a naturally occurring carboxylic acid with chemical formula $\mathrm{CH} 3 \mathrm{CH} 2 \mathrm{COOH}$. It is a clear liquid with pungent odor, molecular formula 74.08, with one carboxylic group. The physical properties is intermediate those smaller carboxylic acids and the largest fatty acids. (http: //en.wikipedia.org/wiki/Propanoic_acid. 2014)

The factors that determine which ligand will coordinate best with a given metal ion are numerous, complicated and not completely understood. (Dodle et al. 1995) The bond between the metal ion and a ligand shows high dependency on the properties of the metal atom. The most important of these properties are the size of metal atom, its ionic charge, atomic number, electronic configuration, electronegativity and ionization potential (William et al. 1985).

Volumetric titrations are usually most conveniently performed with visual indicators. In cases where an indicator is unavailable, potentiometric detection of end point can often be used. Potentiometric titrations are among the most accurate known, because the potential follows actual change in activity and therefore the end point coincide with the equivalent point (Cary D Christian. 1980). Activity means the effective concentration of an ion in the presence of an electrolyte, and it can be used to describe quantitatively the effects of salt on equilibrium constants, and it is also important in potentiometric measurements (Louis Meites. 1981).

\section{Material and methods}

\subsection{Preparation of stock solution}

All materials used in this investigation were chemically pure brand and hence were used without further purification. The solutions used throughout the experiments were prepared in doubly distilled water.

All measurements were carried out with $\mathrm{pH}$-meter Denver instrument ultra-basic $\mathrm{pH} / \mathrm{mV}$ meter with combine electrode at $29 \pm 10 \mathrm{C}$, the sensitivity of the $\mathrm{pH}$-meter is 0.01 unit, the instrument could read $\mathrm{pH}$ in range 0.00 to 14.00 in step 0.01.The $\mathrm{pH}$-meter was 
switched on before half an hour for warm up before starting titration. The instrument was calibrated before each set of titration using two buffer solutions of $\mathrm{pH} 4$ and 10. The electrode was washed with distilled water and dried with tissue paper. The readings were recorded only when the instrument registered a steady value for at least one minute.

\subsection{Procedure}

Three sets of solution total volume of each (V0) $200 \mathrm{~cm} 3$ were prepared for titration against free carbonate sodium hydroxide solution. The change in $\mathrm{pH}$ of solution with each addition of alkali was recorded for each of the following mixtures to calculate values of formation functions $\mathrm{nA}, \mathrm{n}-, \mathrm{pL}$ (where $\mathrm{nA}$ average number of proton associated with ligand, $\mathrm{n}$ - the average number of ligand attached to metal ion and $\mathrm{pL}$ is the free ligand exponential function) (Gurdeep Raj 1986, p.67)

A/ Free acid. (A)

$\mathrm{C} /$ Free acid + ligand. $(\mathrm{A}+\mathrm{L})$

$\mathrm{D} /$ Free acid + ligand + metal ion. $(\mathrm{A}+\mathrm{L}+\mathrm{M})$

All solutions were completed with double distilled water to $200 \mathrm{~cm} 3$, after adding $1 \mathrm{~cm} 3$ of potassium nitrate solution to maintain ionic strength constant, the titrations were carried out in $250 \mathrm{~cm} 3$ beaker with magnet bar inside for stirring the solution. On plotting the observed $\mathrm{pH}$ against the volume of alkali added, different trends were shown, Acid curve (A), a ligand curve $(\mathrm{A}+\mathrm{L})$ lies below the acid curve indicating the dissociation of ligand in the reaction medium, and metal complex curve $(\mathrm{A}+\mathrm{L}+\mathrm{M})$ lies below the ligand titration curve indicating the complex formation. A matlab program has been created for plotting the graph for volumes of alkali solution versus $\mathrm{pH}$ of each three sets of solution (acid, acid+ligand and acid+ligand+metal ion) and secondly to find the volume of alkali required for bringing each one of three sets of solutions to the same $\mathrm{pH}$.

Calvin and Wilson have demonstrated $\mathrm{pH}$ measurement made during titration with alkali solution of ligand in presence and absence of metal ion could be employed to calculate the formation functions $\mathrm{nA}, \mathrm{n}$ - and $\mathrm{pL}$

The values of formation functions were calculated by excel program on personal computer according to the expressions 1,2 and 3 (Minaxi et al. 2011).

$$
{ }^{-}{ }_{A}=y-\frac{\left(V_{1}-V_{2}\right)\left(N^{0}+E^{0}\right)}{\left(V^{0}+V_{1}\right) T_{C L}^{0}}
$$

Where $y=$ the number of dissociable protons, N0 is the concentration of the alkali, E0 is the concentration of the free acid, $T_{C L}^{0}$ is the total ligand concentration, V0 is the total volume of titration solution,V1and V2 the volume of alkali added to acid, and acid +ligand respectively to bring each one to the same $\mathrm{pH}$ value.

The average number of the ligand to metal or metal-ligand formation number at various $\mathrm{pH}$ values determined according to $\mathrm{Ir}-$ ving and Rossotti by the following equation:

$$
n=\frac{-\left(V_{3}-V_{2}\right)\left(E^{0}+N^{0}\right)}{\left(V^{0}+V_{2}\right){ }_{n}{ }_{A}^{T}{ }_{c m}^{0}}
$$

Where V2 and V3 are the volumes of alkali required to mixture of (acid + ligand) and (acid+ligand +metal) to bring them to the same $\mathrm{pH}$ value, $\mathrm{Tcm}$ is the total concentration of the metal and other significances as in equation (1).

A free ligand exponent function (PL) was calculated using equation (3) (Ashwini et al. 2007)

$$
P L=\log _{10}\left\{\frac{1+\beta_{n}^{H} \frac{1}{[\text { anti } \log \mathrm{pH}]^{\mathrm{n}}}}{\mathrm{T}_{\mathrm{CL}}^{0}-{ }_{n T} T_{c m}} \times \frac{\left(V^{0}+V_{3}\right)}{V^{0}}\right\}
$$

Where V3 = volume of alkali required to bring the solution of the complex to same $\mathrm{pH}$ in titration curve.

\section{Result and discussion}

\section{1. proton-ligand stability constant}

\subsubsection{Point-wise calculation method}

The calculation of free ligand exponent function $(\mathrm{pL})$ of metal complexes requires a prior knowledge of the proton-ligand stability constant (Avinash et al. 2013) (equation 3). Using excel program on personal computer the values of $\mathrm{nA}$ at various $\mathrm{pH}$ reading (B) were calculated from the acid and ligand titration curve. For propanoic acid and citric acid the ligand titration curve is well separated from the free acid titration curve at $\mathrm{pH} 2.5$, indicating the dissociation of the ligand in the titration medium.

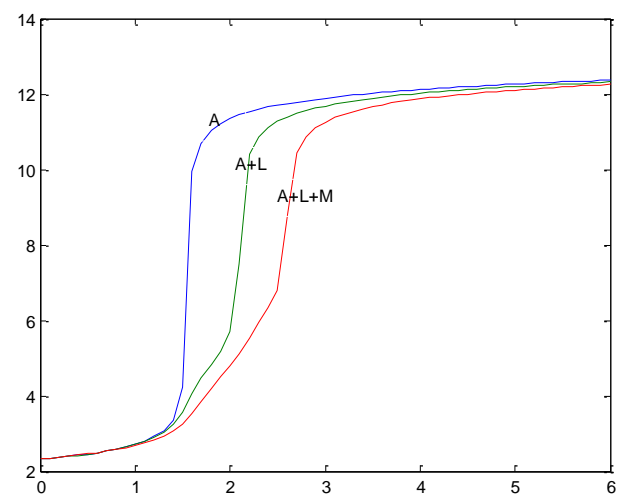

Fig. 1: Titration Curves of Three Sets of Solution. A-Free Acid (HNO3), L-Ligand (Propanoic Acid) and M-Metal Ion ( $\mathrm{Zr}$ )

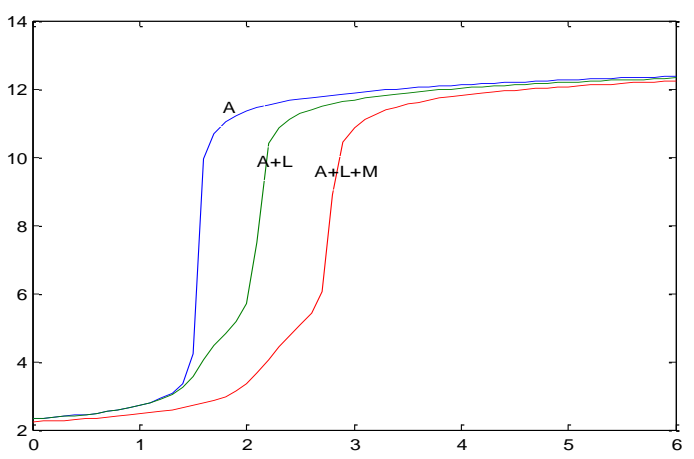

Fig. 2: Titration Curves Of Three Sest Of Solution. A-Free Acid (HNO3), LLigand (Propanoic Acid) And M-Metal Ion (Cr) 


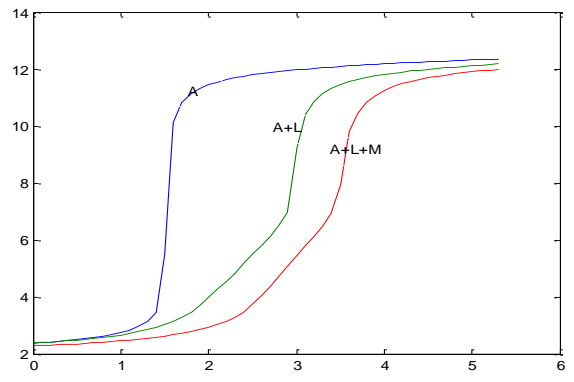

Fig. 3: Titration Curves of Three Sets of Solution. A-Free Acid (HNO3), L-Ligand (Citric Acid) and M-Metal Ion (Zr).

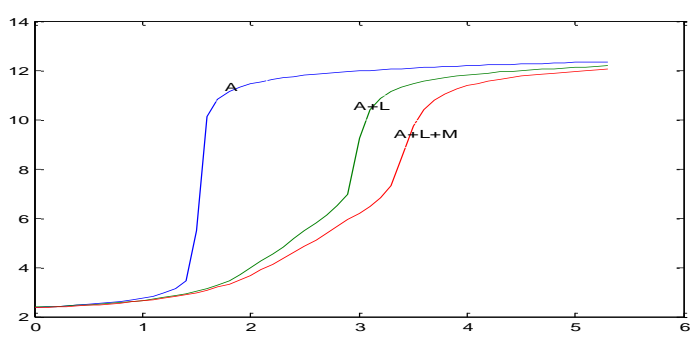

Fig. 4: Titration Curves of Three Sets of Solution. A-Free Acid (HNO3), LLigand ( Citric Acid) and M-Metal Ion (Cr)

According to point-wise calculation method the dissociation constants were calculated on tables below. Values of pKa1, pKa2 and pKa3 were taken as an average on range of 0.2 to $0.8,1.2$ to 1.8 and 2.2 to 2.8 on nA scale respectively (Sayyed et al. 2011).

Table 1: Proton-Ligand Stability Constant of Propionic Acid. Average pka1 $=4.8259$

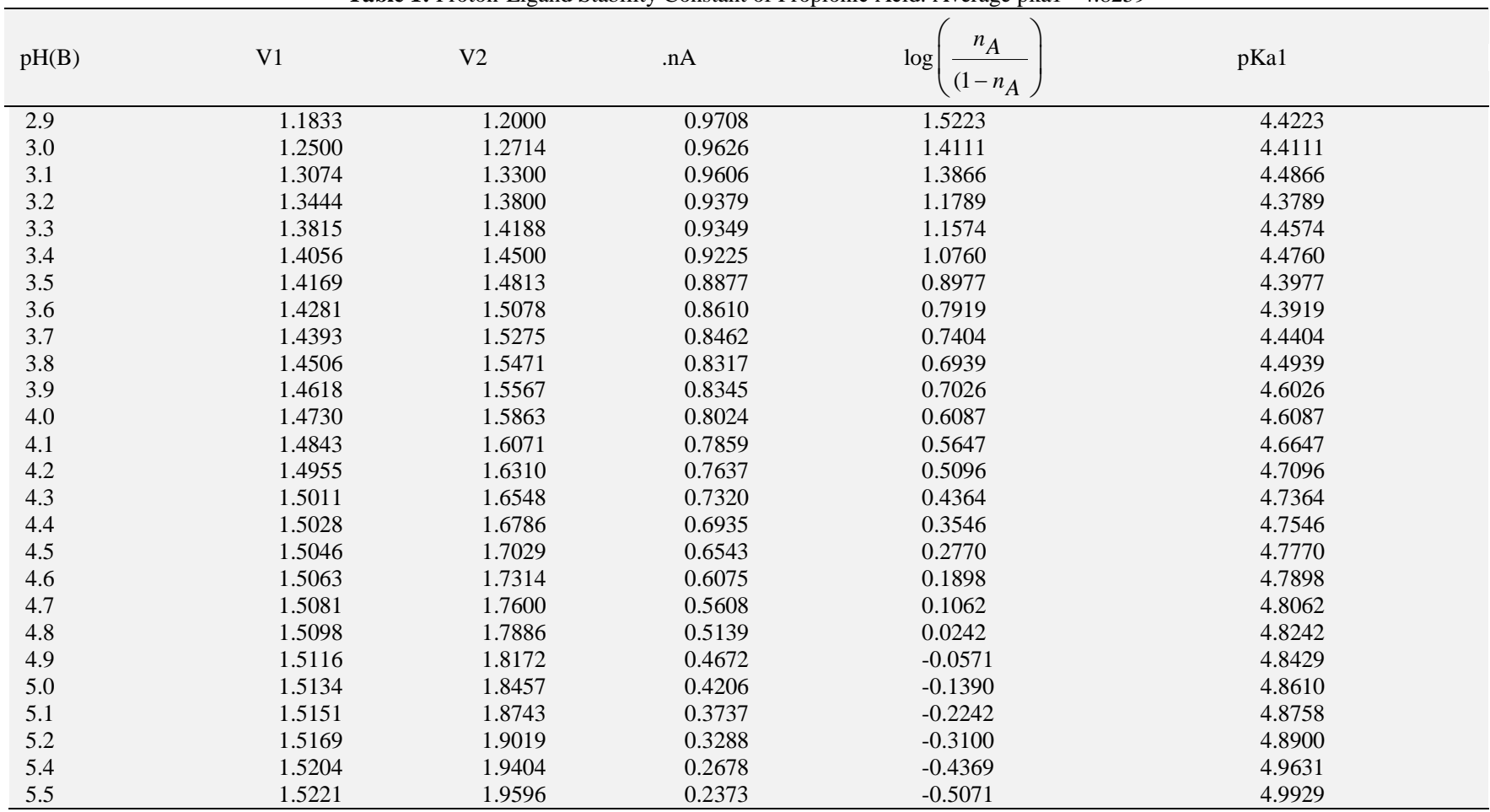

Table 2: First Dissociation Constant of Citric Acid. Average pka1=3.1459

\begin{tabular}{lllllll}
\hline $\mathrm{pH}$ & $\mathrm{nA}$ & $\log \frac{\left(n_{A}-2\right)}{\left(3-n_{A}\right)}$ & $\mathrm{pKa1}$ & $\mathrm{pH}$ & $\mathrm{nA}$ & $\begin{array}{l}\left(n_{A}-2\right) \\
\left(3-n_{A}\right)\end{array}$ \\
\hline 2.5 & 2.8833 & 0.8789 & & & & \\
2.6 & 2.8426 & 0.7287 & 3.3789 & 3.1 & 2.5201 & 0.0349 \\
2.7 & 2.7587 & 0.4975 & 3.3287 & 3.2 & 2.4525 & -0.0828 \\
2.8 & 2.6978 & 0.3635 & 3.1975 & 3.3 & 2.3850 & -0.2034 \\
2.9 & 2.6370 & 0.2443 & 3.1635 & 3.4 & 2.3429 & -0.2825 \\
3.0 & 2.5772 & 0.1353 & 3.1443 & 3.5 & 2.2838 & -0.4021 \\
\hline
\end{tabular}

Table 3: Second Dissociation Constant of Citric Acid. Average pka2 $=4.8270$

\begin{tabular}{|c|c|c|c|c|c|c|c|}
\hline $\mathrm{pH}$ &.$n A$ & $\log \frac{\left(n_{A}-1\right)}{\left(2-n_{A}\right)}$ & $\mathrm{pKa} 2$ & $\mathrm{pH}$ & $\mathrm{nA}$ & $\log \frac{\left(n_{A}-1\right)}{\left(2-n_{A}\right)}$ & pKa2 \\
\hline 4.3 & 1.8275 & 0.5389 & 4.8389 & 5.1 & 1.4364 & -0.1865 & 4.9135 \\
\hline 4.5 & 1.7241 & 0.3105 & 4.8105 & 5.3 & 1.3483 & -0.3633 & 4.9367 \\
\hline 4.6 & 1.6715 & 0.2086 & 4.8086 & 5.4 & 1.3023 & -0.4624 & 4.9376 \\
\hline 4.7 & 1.6178 & 0.1120 & 4.8120 & 5.5 & 1.2564 & -0.5867 & 4.9133 \\
\hline 4.8 & 1.5641 & 0.0330 & 4.8330 & 5.6 & 1.2057 & -0.7365 & 4.8635 \\
\hline 4.9 & 1.5190 & -0.0388 & 4.8612 & 5.7 & 1.1550 & -0.9340 & 4.7660 \\
\hline 5.0 & 1.4777 & -0.1111 & 4.8889 & 5.8 & 1.1043 & -1.2472 & 4.5528 \\
\hline
\end{tabular}


Table 4: Third Dissociation Constant of Citric Acid. Average pka3 $=8.3813$

\begin{tabular}{|c|c|c|c|c|c|c|c|}
\hline $\mathrm{pH}$ & $\mathrm{nA}$ & $\log \frac{n_{A}}{\left(1-n_{A}\right)}$ & $\mathrm{pKa} 3$ & $\mathrm{pH}$ & $\mathrm{nA}$ & $\log \frac{n_{A}}{\left(1-n_{A}\right)}$ & pka3 \\
\hline 6.4 & 0.8159 & 0.6465 & 7.0465 & 8.3 & 0.5630 & 0.1101 & 8.4101 \\
\hline 6.6 & 0.7362 & 0.4458 & 7.0458 & 8.5 & 0.5556 & 0.0970 & 8.5970 \\
\hline 6.7 & 0.7012 & 0.3705 & 7.0705 & 8.6 & 0.5518 & 0.0903 & 8.6903 \\
\hline 6.8 & 0.6662 & 0.3001 & 7.1001 & 8.7 & 0.5480 & 0.0836 & 8.7836 \\
\hline 6.9 & 0.6314 & 0.2337 & 7.1337 & 8.8 & 0.5442 & 0.0769 & 8.8769 \\
\hline 7.0 & 0.6119 & 0.1977 & 7.1977 & 8.9 & 0.5405 & 0.0706 & 8.9706 \\
\hline 7.1 & 0.6082 & 0.1910 & 7.2910 & 9.0 & 0.5367 & 0.0639 & 9.0639 \\
\hline 7.2 & 0.6044 & 0.1841 & 7.3841 & 9.1 & 0.5329 & 0.0573 & 9.1573 \\
\hline 7.3 & 0.6006 & 0.1772 & 7.4772 & 9.2 & 0.5291 & 0.0506 & 9.2506 \\
\hline 7.4 & 0.5968 & 0.1703 & 7.5703 & 9.3 & 0.5223 & 0.0388 & 9.3388 \\
\hline 7.5 & 0.5932 & 0.1638 & 7.6638 & 9.4 & 0.5107 & 0.0186 & 9.4186 \\
\hline 7.6 & 0.5895 & 0.1572 & 7.7572 & 9.5 & 0.4990 & -0.0017 & 9.4983 \\
\hline 7.7 & 0.5855 & 0.1501 & 7.8501 & 9.6 & 0.4874 & -0.0219 & 9.5781 \\
\hline 7.8 & 0.5819 & 0.1436 & 7.9436 & 9.7 & 0.4757 & -0.0422 & 9.6578 \\
\hline 8.0 & 0.5743 & 0.1300 & 8.1300 & 9.9 & 0.4524 & -0.0829 & 9.8171 \\
\hline 8.1 & 0.5705 & 0.1233 & 8.2233 & 10.0 & 0.4408 & -0.1033 & 9.8967 \\
\hline 8.2 & 0.5669 & 0.1168 & 8.3168 & 10.1 & 0.4292 & -0.1237 & 9.9763 \\
\hline
\end{tabular}

\subsection{Metal-ligand stability constant}

\subsubsection{Point-wise calculation method}

Metal titration curve showed a displacement with respect to the ligand titration curve along the volume axis indicating the affinity of ligand with metal ions which release protons and produced the volume difference (V3-V2) (Mane et al. 2011). This is utilized to calculate the values $\mathrm{n}$ - and $\mathrm{pL}$ and further to evaluate the metalligand stability constants. Chromium complexes:

For propanoic acid the variation of $\mathrm{n}$ - is from 0.0260 to 1.9031 , since the ligand is monoprotonic, this indicates the formation of two complex species ML1 and ML2. For tribasic ligand citric acid, the values of $n$ - extend between 0.0633 and 2.9925 , indicate formation of ML chelate.

Zirconium complexes

For propanoic acid n- extends in the range 2.4881 to 2.9968 this indicates the formation of 1:3 complex only. For citric acid The n's values extend between 1.0892 to 2.8752 , indicating formation of $1: 1$ chelate only because the ligand is tribasic.

Table 5: Point-Wise Calculation Method Cr-Propanoate Logk1 (Average Value $=4.5499$ )

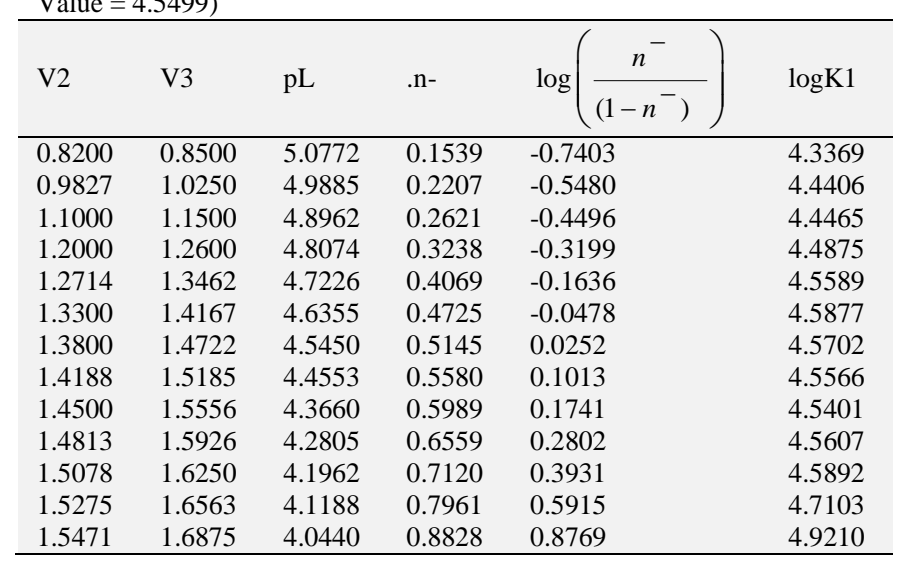

Table 6: Point-Wise Calculation Method Cr-Propionate Logk2 (Average Value $=3.6038$ )

\begin{tabular}{llllll}
\hline V2 & V3 & pL & $\mathrm{n}$ & $\log \left(\frac{\left(n^{-}-1\right)}{\left(2-n^{-}\right)}\right)$ & $\operatorname{logK2}$ \\
\hline 1.6310 & 1.8030 & 3.7624 & 1.1772 & -0.6667 & 3.0957 \\
1.6548 & 1.8333 & 3.7073 & 1.2745 & -0.4220 & 3.2853 \\
1.6786 & 1.8636 & 3.6637 & 1.3942 & -0.1867 & 3.4770 \\
1.7029 & 1.8939 & 3.6304 & 1.5255 & 0.04436 & 3.6748 \\
1.7314 & 1.9286 & 3.6185 & 1.6959 & 0.35958 & 3.9780 \\
\hline
\end{tabular}

Table 7: Point-Wise Calculation Method Cr-Citrate Logk1 (Average Val$\mathrm{ue}=6.1327$ )

\begin{tabular}{llllll}
\hline & & & & & \\
$\mathrm{V} 2$ & $\mathrm{~V} 3$ & $\mathrm{n}-$ & $\mathrm{pL}$ & $\log \frac{n^{-}}{\left(1-n^{-}\right)}$ & $\operatorname{logK1}$ \\
& & & & & \\
\hline 1.7611 & 1.8438 & 0.1844 & 8.7155 & -0.6457 & 8.0697 \\
1.8130 & 1.9053 & 0.2111 & 8.4197 & -0.5726 & 7.8471 \\
1.8565 & 1.9579 & 0.2389 & 8.1242 & -0.5033 & 7.6209 \\
1.9000 & 2.0091 & 0.2650 & 7.8284 & -0.4430 & 7.3854 \\
1.9370 & 2.0546 & 0.2932 & 7.5330 & -0.3820 & 7.1510 \\
1.9741 & 2.1000 & 0.3225 & 7.2379 & -0.3223 & 6.9156 \\
2.0104 & 2.1455 & 0.3556 & 6.9434 & -0.2581 & 6.6853 \\
2.0448 & 2.1909 & 0.3948 & 6.6500 & -0.1856 & 6.4644 \\
2.0793 & 2.2333 & 0.4275 & 6.3557 & -0.1269 & 6.2288 \\
2.1138 & 2.2750 & 0.4600 & 6.0615 & -0.0696 & 5.9918 \\
2.1483 & 2.3167 & 0.4945 & 5.7678 & -0.0096 & 5.7582 \\
2.1828 & 2.3583 & 0.5306 & 5.4748 & 0.0533 & 5.5282 \\
2.2179 & 2.4000 & 0.5678 & 5.1828 & 0.1186 & 5.3013 \\
2.2536 & 2.4400 & 0.6004 & 4.8911 & 0.1769 & 5.0680 \\
2.2893 & 2.4800 & 0.6353 & 4.6023 & 0.2410 & 4.8433 \\
2.3200 & 2.5192 & 0.6832 & 4.3206 & 0.3337 & 4.6543 \\
2.3486 & 2.5577 & 0.7371 & 4.0487 & 0.4477 & 4.4964 \\
2.3772 & 2.5962 & 0.7940 & 3.7934 & 0.5861 & 4.3795 \\
\hline
\end{tabular}

Table 8: Point-Wise Calculation Method Zr-Propanoate Logk3 (Average Value 11.5165)

\begin{tabular}{llllll}
\hline V2 & V3 & .n- & .pL & $\log \frac{\left(n^{-}-2\right)}{\left(3-n^{-}\right)}$ & $\operatorname{logK3}$ \\
\hline 0.3333 & 0.8000 & 2.4881 & 11.4807 & -0.0207 & 11.4661 \\
0.6200 & 1.1000 & 2.4974 & 11.3885 & -0.0045 & 11.3883 \\
0.820 & 1.3167 & 2.5456 & 11.3325 & 0.0795 & 11.4156 \\
0.9827 & 1.4833 & 2.6101 & 11.2990 & 0.1944 & 11.4962 \\
1.1000 & 1.6222 & 2.7356 & 11.3686 & 0.4443 & 11.8160 \\
\hline
\end{tabular}

\subsubsection{Half integral method}

If a graph is plotted between $\mathrm{n}^{-}$and $\mathrm{pL}$, then the corresponding values of $\mathrm{pL}$ at $\mathrm{n}^{-}$equal to 0.5 and 1.5 gives $\operatorname{logK} 1$ and $\log \mathrm{K} 2$ respectively (Gurdeep Raj 1986, p.67).

Generally for $\log \mathrm{Kn}$ the value of $\mathrm{pL}$ at which $\mathrm{n}^{-}=\mathrm{n}-0.5$ corresponds to $\operatorname{logKn}$. Other expression for this 
Table 9: Point-Wise Calculation Method Zr-Citrate Logk1 (Average Value 7.5967)

\begin{tabular}{llllll} 
& & & & & \\
V2 & V3 & $n-$ & pL & $\log \frac{\left(n^{-}-1\right)}{\left(2-n^{-}\right)}$ & \\
& & & & & \\
& & & & & \\
1.4700 & 2.0700 & 1.2179 & 10.1245 & -0.5549 & 9.5696 \\
1.5583 & 2.1636 & 1.2560 & 9.8341 & -0.4633 & 9.3708 \\
1.6357 & 2.2400 & 1.2880 & 9.5423 & -0.3930 & 9.1492 \\
1.7056 & 2.3056 & 1.3146 & 9.2492 & -0.3383 & 8.9109 \\
1.7611 & 2.3611 & 1.3378 & 8.9554 & -0.2922 & 8.6631 \\
1.8130 & 2.4111 & 1.3678 & 8.6634 & -0.2353 & 8.4281 \\
1.8565 & 2.4482 & 1.3939 & 8.3705 & -0.1871 & 8.1834 \\
1.9000 & 2.4852 & 1.4215 & 8.0781 & -0.1375 & 7.9406 \\
1.9370 & 2.5188 & 1.4508 & 7.7863 & -0.0858 & 7.7005 \\
1.9741 & 2.5500 & 1.4754 & 7.4933 & -0.0429 & 7.4505 \\
2.0104 & 2.5813 & 1.5028 & 7.2013 & 0.0049 & 7.2062 \\
2.0448 & 2.6125 & 1.5339 & 6.9105 & 0.0590 & 6.9695 \\
2.0793 & 2.6438 & 1.5669 & 6.6205 & 0.1169 & 6.7375 \\
2.1138 & 2.6750 & 1.6014 & 6.3314 & 0.1787 & 6.5101 \\
2.1483 & 2.7061 & 1.6379 & 6.0432 & 0.2459 & 6.2891 \\
2.1828 & 2.7364 & 1.6739 & 5.7555 & 0.3152 & 6.0708 \\
2.2179 & 2.7667 & 1.7113 & 5.4692 & 0.3917 & 5.8609 \\
2.2536 & 2.7970 & 1.7504 & 5.1850 & 0.4781 & 5.6631 \\
2.2893 & 2.8257 & 1.7868 & 4.9026 & 0.5672 & 5.4698 \\
\hline
\end{tabular}

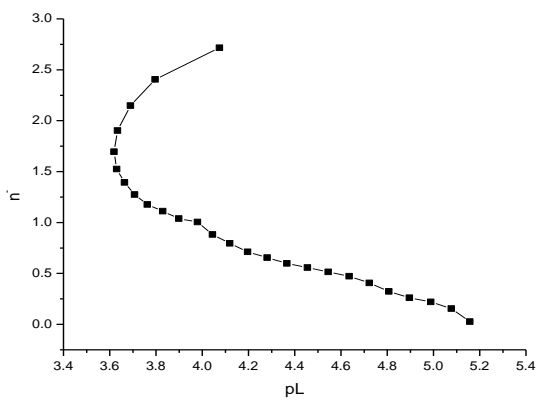

Fig. 5: Chromium Propanoate $\operatorname{Logk} 1=4.54$ and $\operatorname{Logk} 2=3.632$ (Half Integral Method)

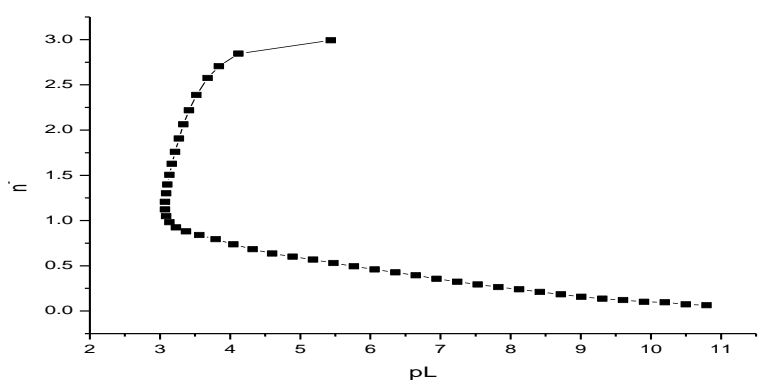

Fig. 6: Chromium Citrate Logk1=5.72 (Half Integral Method

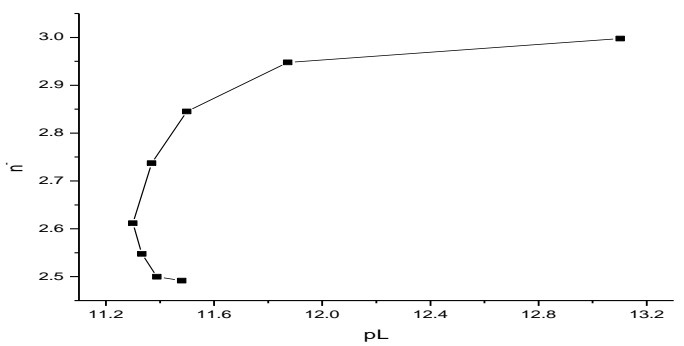

Fig. 7: Zirconium Propanoiate Logk3=11.382

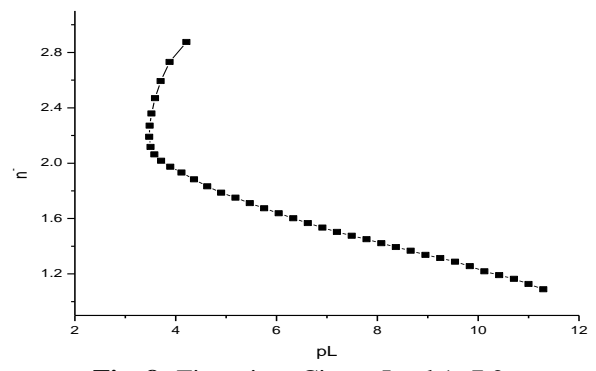

Fig. 8: Zirconium Citrate Logk1=7.2
$K_{n}=\left(\frac{1}{[\mathrm{~L}]}\right)_{\text {at } \mathrm{n}^{-}=\mathrm{n}-0.5}$

\subsubsection{Linear plot method:}

Plotting $\log (\mathrm{n} / \mathrm{n}-1)$ and $\log (\mathrm{n}-1) /(2-\mathrm{n})$ versus corresponding $\mathrm{pL}$ gives $\log \mathrm{K} 1$ and $\log \mathrm{K} 2$ respectively (Anjani et al. 2011).

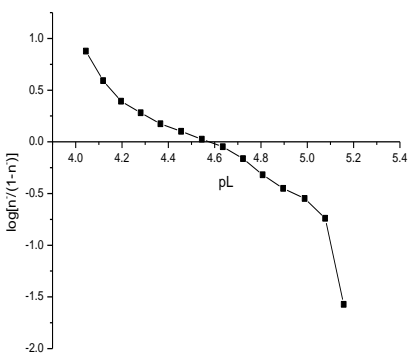

Fig. 9: Chromium Propanoate Logk1=4.626 (Linear Plot Method)

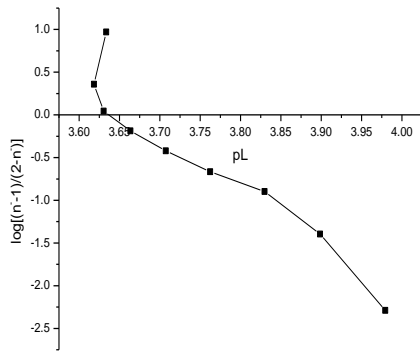

Fig. 10: Chromium Propanoate Logk2=3.63 (Linear Plot Method)

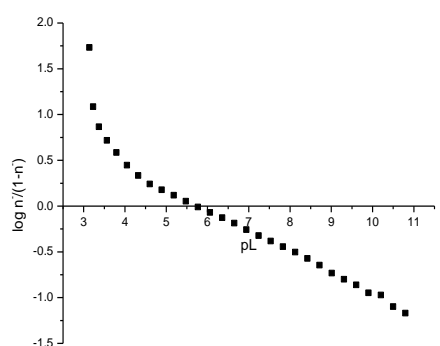

Fig. 11: Chromium Citrate Logk1=5.72 (Linear Plot Method)

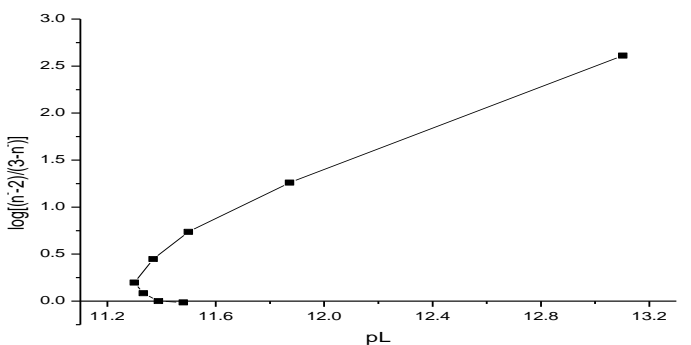

Fig. 12: Zirconium Propanoate Logk3=11.382 (Linear Plot Method)

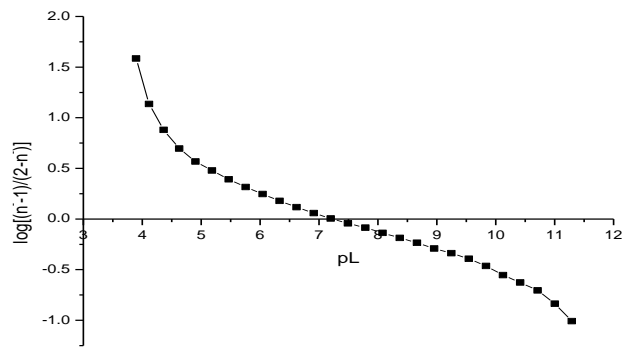

Fig. 13: Zirconium Citrate Logk1=7.41 (Linear Plot Method)

\subsubsection{Least squares method}

For estimation of $\mathrm{K} 2$ and $\mathrm{K} 3$ for chromium propanoiate and chromium citrate by this method, linear equation of Rossotti and Rossotti as expressed below has been employed: 
$n^{-}=\left(1-n^{-}\right)[L] K_{1}+\left(2-n^{-}\right)[L] K_{1} K_{2}$

This on division by (1-n-) [L] K1 and further rearranging becomes:

$$
\frac{n^{-}}{\left(1-n^{-}\right)[L]}=\frac{\left(2-n^{-}\right) *[L]}{\left(1-n^{-}\right)} \times K_{1} K_{2}+K_{1}
$$

Table 10: Determination of Chromium Propanoiate Stability Constants by Least Squares Method (Averg. Value Logk1=4.2198 and Logk2 (Average Value $=3.7344$ )

\begin{tabular}{|c|c|c|c|c|}
\hline $\mathrm{pL}$ & $\mathrm{n}$ & [L] & $\frac{\left(2-n^{-}\right)[L]}{\left(1-n^{-}\right)}$ & $\frac{n^{-}}{\left(1-n^{-}\right)[L]^{2}}$ \\
\hline 5.1571 & 0.0260 & $6.9647 \mathrm{E}-06$ & $1.4116 \mathrm{E}-05$ & 3838.8930 \\
\hline 5.0772 & 0.1539 & $8.3716 \mathrm{E}-06$ & $1.8266 \mathrm{E}-05$ & 21723.1864 \\
\hline 4.9885 & 0.2207 & $1.0267 \mathrm{E}-05$ & $2.3442 \mathrm{E}-05$ & 27579.1226 \\
\hline 4.8962 & 0.2621 & $1.2701 \mathrm{E}-05$ & $2.9912 \mathrm{E}-05$ & 27960.5246 \\
\hline 4.8074 & 0.3238 & $1.5583 \mathrm{E}-05$ & $3.8626 \mathrm{E}-05$ & 30723.2399 \\
\hline 4.7226 & 0.4069 & $1.8943 \mathrm{E}-05$ & $5.0881 \mathrm{E}-05$ & 36218.9184 \\
\hline 4.6355 & 0.4725 & $2.3148 \mathrm{E}-05$ & $6.7031 \mathrm{E}-05$ & 38701.2932 \\
\hline 4.5450 & 0.5145 & $2.8513 \mathrm{E}-05$ & $8.7244 \mathrm{E}-05$ & 37171.0648 \\
\hline 4.4553 & 0.5580 & $3.5049 \mathrm{E}-05$ & 0.00014351 & 36023.8284 \\
\hline 4.3660 & 0.5989 & 4.3056E-05 & 0.0002 & 34678.2783 \\
\hline 4.2805 & 0.6559 & $5.2416 \mathrm{E}-05$ & 0.0002 & 36368.7292 \\
\hline 4.1962 & 0.7120 & $6.3656 \mathrm{E}-05$ & 0.0003 & 38837.3431 \\
\hline 4.1188 & 0.7961 & 7.6076E-05 & 0.0004 & 51320.6695 \\
\hline 4.0440 & 0.8828 & $9.0363 \mathrm{E}-05$ & 0.0009 & 83359.2057 \\
\hline 3.9795 & 1.0051 & 0.0001 & -0.0203 & -1870517.592 \\
\hline 3.8297 & 1.1123 & 0.0001 & -0.0012 & -66924.1810 \\
\hline 3.7624 & 1.1772 & 0.0002 & -0.0008 & -38434.2168 \\
\hline 3.7073 & 1.2745 & 0.0002 & -0.0005 & -23662.7599 \\
\hline 3.6637 & 1.3942 & 0.0002 & -0.0003 & -16303.5508 \\
\hline 3.6304 & 1.5255 & 0.0002 & -0.0002 & -12394.8823 \\
\hline 3.6185 & 1.6959 & 0.0002 & -0.0001 & -10122.9771 \\
\hline 3.6337 & 1.9031 & 0.0002 & $-2.4942-05$ & -9066.0208 \\
\hline 3.6895 & 2.1486 & 0.0002 & 2.6452E-05 & -9150.3771 \\
\hline
\end{tabular}

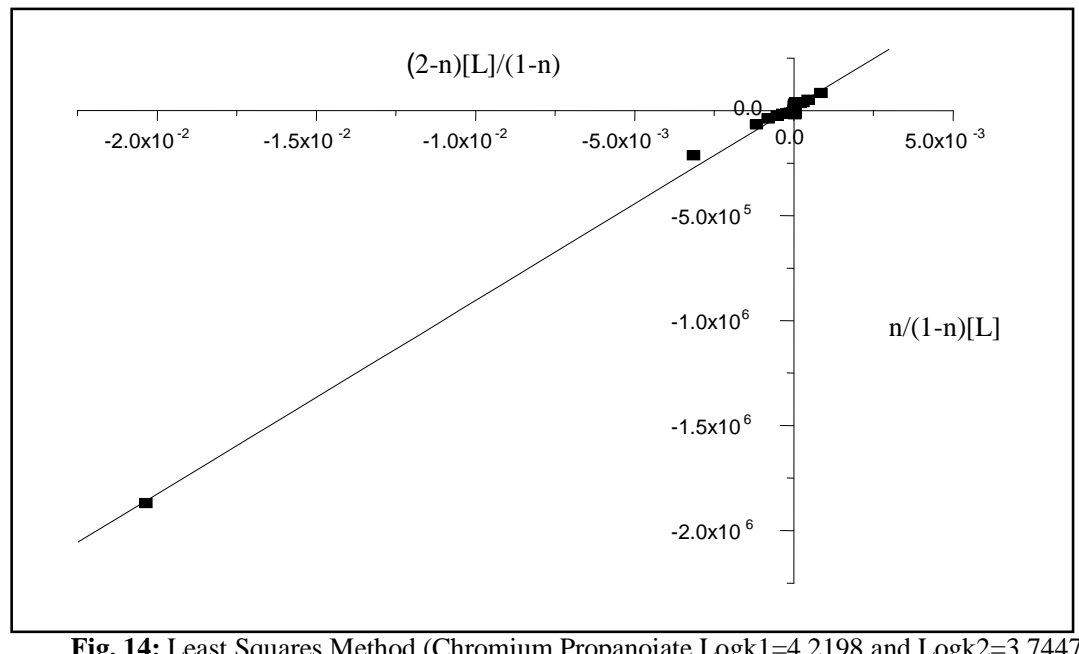

Linear Regression for Data1_B:

$\mathrm{Y}=\mathrm{A}+\mathrm{B} * \mathrm{X}$

\begin{tabular}{llll}
\hline Parameters & Value & Error & R2 \\
\hline Intercept & 16590.2608 & 4192.90636 & 0.99849 \\
Slope & $9.2152 \mathrm{E} 7$ & $1.03405 \mathrm{E} 6$ & \\
\hline
\end{tabular}

$\mathrm{R} 2$ is coefficient of determination. This is the famous number people quote to prove how good the fit is. It is quite useless for evaluating a working curve unless you count the "number of nines" you get. (0.978 is a pretty bad working curve. 0.999 is probably a good one.) For estimation of chromium citrate stability constant by this method, and because only one complex is formed, equation familiar as Henderson or Hasselbalch's equation was employed as follows: (Rossotti et al. 1955).

$$
\frac{n^{-}}{\left(1-n^{-}\right)}=\beta_{1}[L] \quad \log \frac{n^{-}}{\left(1-n^{-}\right)}=\log \beta_{1}+\log [L]
$$


Table 11: Determination of Chromium Citrate Stability Constant by Hasselbach's Equation. Logk1 (Average. Value =6.0509)

\begin{tabular}{|c|c|c|c|c|c|c|c|c|c|}
\hline $\mathrm{pL}$ & $\mathrm{n}$ & $\log [\mathrm{L}]$ & $\log \frac{n^{-}}{\left(1-n^{-}\right)}$ & $\log \frac{n^{-}}{\left(1-n^{-}\right)}-\log [L]$ & $\mathrm{pL}$ & $\mathrm{n}$ & $\log [\mathrm{L}]$ & $\log \frac{n^{-}}{\left(1-n^{-}\right)}$ & $\log \frac{n^{-}}{\left(1-n^{-}\right)}-\log [L]$ \\
\hline 11.7244 & 0.2634 & -11.7244 & 0.2796 & 12.0040 & 5.4748 & -5.4748 & -5.4748 & 0.2517 & 5.7265 \\
\hline 11.4032 & 0.1214 & -11.4032 & 0.2918 & 11.6950 & 5.1828 & -5.1828 & -5.1828 & 0.2471 & 5.4299 \\
\hline 11.0994 & 0.0923 & -11.0994 & 0.2941 & 11.3935 & 4.8911 & -4.8911 & -4.8911 & 0.2430 & 5.1341 \\
\hline 10.7956 & 0.0633 & -10.7956 & 0.2963 & 11.0919 & 4.6023 & -4.6023 & -4.6023 & 0.2384 & 4.8407 \\
\hline 10.4975 & 0.0740 & -10.4975 & 0.2955 & 10.7930 & 4.3206 & -4.3206 & -4.3206 & 0.2318 & 4.5523 \\
\hline 9.9022 & 0.1015 & -9.9022 & 0.2934 & 10.1955 & 3.7934 & -3.7934 & -3.7934 & 0.2149 & 4.0083 \\
\hline 9.6053 & 0.1212 & -9.6053 & 0.2918 & 9.8971 & 3.5624 & -3.5624 & -3.5624 & 0.2073 & 3.7697 \\
\hline 9.3079 & 0.1371 & -9.3079 & 0.2905 & 9.5984 & 3.3701 & -3.3701 & -3.3701 & 0.1999 & 3.5701 \\
\hline 9.0111 & 0.1562 & -9.0110 & 0.2889 & 9.2999 & 3.2277 & -3.2277 & -3.2277 & 0.1917 & 3.4193 \\
\hline 8.7155 & 0.1844 & -8.7155 & 0.2866 & 9.0020 & 3.1378 & -3.1378 & -3.1378 & 0.1800 & 3.3178 \\
\hline 8.4197 & 0.2111 & -8.4197 & 0.2843 & 8.7040 & 3.0903 & -3.0903 & -3.0903 & 0.1653 & 3.2556 \\
\hline 8.1242 & 0.2389 & -8.1242 & 0.2818 & 8.4060 & 3.0723 & -3.0723 & -3.0723 & 0.1469 & 3.2192 \\
\hline 7.8284 & 0.2650 & -7.8284 & 0.2795 & 8.1079 & 3.0738 & -3.0738 & -3.0738 & 0.1228 & 3.1966 \\
\hline 6.9434 & 0.3556 & -6.9434 & 0.2708 & 7.2142 & 3.1356 & -3.1356 & -3.1356 & -0.0027 & 3.1328 \\
\hline 6.6500 & 0.3948 & -6.6500 & 0.2668 & 6.9168 & 3.1715 & -3.1715 & -3.1715 & -0.0892 & 3.0823 \\
\hline 6.3557 & 0.4275 & -6.3557 & 0.2634 & 6.6190 & 3.2149 & -3.2149 & -3.2149 & -0.2356 & 2.9793 \\
\hline 6.0615 & 0.4600 & -6.0615 & 0.2598 & 6.3213 & 3.2692 & -3.2692 & -3.2692 & -0.5897 & 2.6795 \\
\hline 5.7678 & 0.4945 & -5.7678 & 0.2559 & 6.0237 & - & - & - & - & - \\
\hline
\end{tabular}

Since only one complex zirconium propanoiate is formed Henderson's equation is employed.

Table 12: Determination of Zirconium Propanoiate Stability Constant by Hasselbach's Equation Logk3 (Average Value =11.5124)

\begin{tabular}{|c|c|c|c|c|c|c|c|c|c|}
\hline $\mathrm{pL}$ & $\mathrm{n}$ & $\log [L]$ & $\log \frac{n^{-}}{\left(1-n^{-}\right)}$ & $\log \frac{\left(n^{-}-2\right)}{\left(3-n^{-}\right)}-\log [L]$ & $\mathrm{pL}$ & $\mathrm{n}$ & $\log [\mathrm{L}]$ & $\log \frac{\left(n^{-}-2\right)}{\left(3-n^{-}\right)}$ & $\log \frac{\left(n^{-}-2\right)}{\left(3-n^{-}\right)}-\log [L]$ \\
\hline 11.4817 & 2.4881 & -11.4807 & -0.0207 & 11.4600 & 11.3686 & 2.7356 & -11.3686 & 0.44434 & 11.8129 \\
\hline 11.3325 & 2.5456 & -11.3325 & 0.07949 & 11.4120 & 11.8720 & 2.9470 & -11.8720 & 1.25219 & 13.1242 \\
\hline 11.2990 & 2.6101 & -11.2990 & 0.19436 & 11.4934 & 13.1023 & 2.9968 & -13.1023 & 2.49601 & 15.5984 \\
\hline
\end{tabular}

Table 13: Determination of Zirconium Citrate Stability Constant, by Hasselbach's Equation Logk1 (Average Value =7.5152)

\begin{tabular}{|c|c|c|c|c|c|c|c|c|c|}
\hline $\mathrm{pL}$ & $\mathrm{n}$ & $\log [L]$ & $\log \frac{n^{-}}{\left(1-n^{-}\right)}$ & $\log \frac{n^{-}}{\left(1-n^{-}\right)}-\log [L]$ & $\mathrm{pL}$ & $\mathrm{n}$ & $\log [\mathrm{L}]$ & $\log \frac{n^{-}}{\left(1-n^{-}\right)}$ & $\log \frac{n^{-}}{\left(1-n^{-}\right)}-\log [L]$ \\
\hline 11.2928 & 1.0892 & -11.2928 & 1.0868 & 12.3797 & 6.04322 & 1.6379 & -6.0432 & 0.4095 & 6.4528 \\
\hline 10.7112 & 1.1649 & -10.7112 & 0.8491 & 11.5603 & 5.46924 & 1.7113 & -5.4692 & 0.3813 & 5.8505 \\
\hline 10.4177 & 1.1910 & -10.4177 & 0.7949 & 11.2127 & 5.18504 & 1.7504 & -5.1850 & 0.3678 & 5.5529 \\
\hline 10.1245 & 1.2179 & -10.1245 & 0.7472 & 10.8718 & 4.90260 & 1.7868 & -4.9026 & 0.3562 & 5.2588 \\
\hline 9.83406 & 1.2560 & -9.8341 & 0.6907 & 10.5248 & 4.62838 & 1.8324 & -4.6284 & 0.3427 & 4.9711 \\
\hline 9.54227 & 1.2880 & -9.5423 & 0.6505 & 10.1928 & 4.36531 & 1.8834 & -4.3653 & 0.3288 & 4.6941 \\
\hline 9.24920 & 1.3146 & -9.2492 & 0.6210 & 9.8703 & 4.11750 & 1.9318 & -4.1175 & 0.3166 & 4.4341 \\
\hline 8.95536 & 1.3378 & -8.9554 & 0.5977 & 9.5531 & 3.89417 & 1.9746 & -3.8942 & 0.3066 & 4.2008 \\
\hline 8.66337 & 1.3678 & -8.6634 & 0.5704 & 9.2338 & 3.71066 & 2.0177 & -3.7107 & 0.2972 & 4.0079 \\
\hline 8.37046 & 1.3939 & -8.3705 & 0.5488 & 8.9193 & 3.57813 & 2.0635 & -3.5781 & 0.2879 & 3.8660 \\
\hline 8.07807 & 1.4215 & -8.0781 & 0.5280 & 8.6060 & 3.50019 & 2.1173 & -3.5002 & 0.2776 & 3.7778 \\
\hline 7.78627 & 1.4508 & -7.7863 & 0.5077 & 8.2939 & 3.47490 & 2.1909 & -3.4749 & 0.2647 & 3.7396 \\
\hline 7.49330 & 1.4754 & -7.4933 & 0.4919 & 7.9852 & 3.48490 & 2.2715 & -3.4849 & 0.2520 & 3.7369 \\
\hline 7.20129 & 1.5028 & -7.2013 & 0.4755 & 7.6768 & 3.52143 & 2.3589 & -3.5214 & 0.2395 & 3.7610 \\
\hline 6.62055 & 1.5669 & -6.6205 & 0.4415 & 7.0621 & 3.70390 & 2.5931 & -3.7039 & 0.2116 & 3.9155 \\
\hline 6.33138 & 1.6014 & -6.3314 & 0.4253 & 6.7567 & 3.88073 & 2.7308 & -3.8807 & 0.1980 & 4.0788 \\
\hline
\end{tabular}

\section{Conclusion}

The three graphical method half integral method, linear plot method and least squares method in addition to the two algebraic ones (point-wise and Henderson's equation) give nearly the same results, but in using least squares method for determination of chromium-propanoic acid which forms 1:1 and 1:2 complexes, the results obtained were slightly different specially in $\mathrm{Kn}-1$, where the error accumulated, this method has many restriction which can be found in literature.

Citric acid forms only one chelate with chromium and zirconium (1:1). Propanoic acid forms $1: 1$ and 1:2 complexes with chromium and with zirconium forms a 1:3 complex only.

Reader no doubt by now may ask the question "what method can I use, and which should give me the most accurate value". Each of these methods employed above seen satisfactorily to give the an- swer, with difference $\leq 0.3$, for a system where $\mathrm{N} \leq 3$ ( $\mathrm{N}$ is the possible coordination sites).

The most important thing in this work that matlab program is used for determination of the volume of alkali that required to bring the three sets of solution to the same $\mathrm{pH}$, which is important for determination of formation functions $\mathrm{nA}, \mathrm{n}$ - and $\mathrm{pL}$.

Interaction of metal ion with carboxylic acid shows high dependency on the salt that added to maintain the ionic strength of the medium, so potassium nitrate with nitric acid as free acid is adequate, for nitrate ion has very slight complexing tendency and the competition between nitrate ion and the ligands under study is of minor importance. 


\section{References}

[1] Sanjay Ubale, N.G. Palaskar, Nazahar Farooqui. 2002. Effect of temperature and media on complexation of Sm (III) with 5-bromo-2hydroxy Acetophenone. 14, (1), 99-102.

[2] Santosh, D. Deosarkar, Ashok, B. Kalambe, Vijay, J. Thakare, Shivraj, G. Wanale, Vinay, V. Hiwase. 2011. Potentiometric studies of electrolyte effects on complex equilibria of some substituted 5-(2-hydroxy phenyl) Pyrazoles. Der pharma chemic. 3, (6), 75-83.

[3] Sumer, D. Thakur, Devidas, T. Mahajan, Kamlesh, P. Munot, Rajesh D. Deshmukh, Mangesh, S. Tihile. 2011b. Metal-ligand stability constants of Th (III), Sm (III), Nd (III) and Pr (III) metal ion complexes with two mercapto-4-substituted phenyl-6-substituted phenyl prymidines at $0.1 \mathrm{M}$ ionic strength $\mathrm{pH}$-metrically. Der pharma chem. 3, (6), 382-389.

[4] Abdulbaset, A. Zaid, Mazahar, Farooqui, D.M. Janrao. 2012. Study of stability constant of biological active molecules (drug) using potentiometric titration technique. J. of chem. boils. phys. Sc. 2, (1), 67-81.

[5] F.R.,'Hartley, C. Burgess, R.M. Alcock.1980. Solution equilibria, first ed. John Wiley and sons, New York.

[6] Cotton and Wilkinson, 1972, advanced inorganic chemistry, 3rd edition, John wiley \& sons.

[7] Boner and Pardue, 1996, Chem. Experimental science, 2nd edition, John wiley \& sons Inc.

[8] http://en.wikipedia.org/wiki/Citric_acid

[9] http://en.wikipedia.org/wiki/Propanoic_acid

[10]Dodle Douglas, Darl H. Daniel, 1995, Concept and model of inorganic chem. 2nd edition, Xerox College united states.

[11]William L. Masterton and others, 1985, Chemical principles, 6th edition, CBS college publishing.

[12]Cary D. Christian, 1980. Analytical chemistry, John wiley and sons,Inc.

[13]Louis Meites,1981.An introduction to chemical equilibrium and kinetics, first edition, Pergamon press

[14]E-book: Gurdeep, Raj, 1986.Advance inorganic chemistry- II , first ed. Satyendra Rastogi "Mitra" p.67.Book code : 277-12 Ninth thoroughly revised 2004Twelfth ed. 2010

[15]Minaxi, Maru, M.K. Shah. 2011. Synthesis and physic-chemical studies of some divalent transition metal complexes of 2-(2'-chloro-6'substituted quinoliny)-1H-benzo[d]imidazole ligands. J. Chem. Res. 2, (2), 14-27.

[16]Ashwini, kumar, Ashok, Kumar, Malik. 2007. Potentiometric studies on the complexes of $\mathrm{Cu}$ ( II ), $\mathrm{Cd}$ ( II ), $\mathrm{Co}$ ( II ), Ni ( II ), Pb ( II ), and Zn ( II ) with Gabapentin. Euras. J. analy. Chem. 2, (2), 1-6.

[17]Avinash, Ramteke, Marutil, Narwade. 2013. Studies of stability constants of complexes of chlorosubstituted pyrazoles and pyrazoline with $\mathrm{Cu}$ ( II ), Ni (II), Co ( II) and Nd (II) metal ions in 70\% dioxane- water mixture at 0.1 ionic strength. Arch. of appl. Sc. 5, (1) 231-237.

[18]Sayyed, Hussain, Abdul Rahim, Mazahar, Farooqui. 2011. Studies of binary complexes of metal ions with mandelic acid by potentiometry. Chem. J. 02, (6), 206-209.

[19]G. V., Mane, L.P. shinde, D.G. Kolhatkar. 2011. pH-metric study of proton-ligand and metal-ligand stability constant complexes of transition and inner transition metal ions with some organic acids.Inter. J. Chemistry. 3, (2), 181-187.

[20]Anjani, Kumar, Mishra, Prem, Mohan, Mishra, Sunil, K. Misra. 2011. Equilibrium studies on bivalent transition metal chelates with biologically active schiff's base of heterocyclic aldehydes. Ras. J. Chem. 4 (2), 303-319.

[21]F. J. C. Rossotti, H. S. Rossotti. 1955. Graphical methods for determining equilibrium constants.1- system of mononuclear complexes. Ac. chem. scandinavica. 9, 1166-1176. 